\title{
Multicolor photometry of ten Seyfert 1 galaxies
}

\author{
N. V. Boris ${ }^{1}$, C. J. Donzelli ${ }^{1}$, M. G. Pastoriza ${ }^{2, \star}$, A. Rodriguez-Ardila $^{3, \star \star \star \star}$, and D. L. Ferreiro ${ }^{1}$ \\ 1 IATE, Observatorio Astronómico, Universidad Nacional de Córdoba, Laprida 854, 5000, Córdoba, Argentina \\ 2 Departamento de Astronomia - UFRGS. Av. Bento Gonçalves 9500, CEP 91501-970, Porto Alegre, RS, Brazil \\ 3 Instituto Astronômico e Geofísico - Universidade de São Paulo, Av. Miguel Stefano 4200, CEP 04301-904, \\ São Paulo, SP, Brazil
}

Received 6 September 2001 / Accepted 7 December 2001

\begin{abstract}
We present new valuable $B V I$ photometry of ten Seyfert 1 galaxies and narrow band $\mathrm{H} \alpha$ images for six of these objects. The results indicate that the distribution of the luminosity of the sample has an amplitude of almost $4 \mathrm{mag}$ with an average of $M_{B}=-20.7$. The observed morphologies are confined to early type galaxies. A barred structure is found in only 2 objects. Despite that early morphological types are dominant in this sample, integrated $(B-V)$ colors are very blue. For instance, the SO galaxies show, on average, a $(B-V)=0.78$. This effect seems to be caused by the luminosity contribution of the active nucleus and/or the disk to the total luminosity of the galaxy. In the $B$ band, the contribution of the active galactic nucleus to the total luminosity of the galaxy varies from $3 \%$ to almost $60 \%$ and the bulge to disk luminosity ratio $\left(L_{\text {bulge }} / L_{\text {disk }}\right)$ ranges from 0.6 to 22. Signs of tidal interactions seem to be a common characteristic since they are observed in 6 of the objects and one of them seems to be located in a poor cluster not yet identified in the literature. In contrast, H $\alpha$ extended emission is rare, with only 1 galaxy showing clear evidence of it. Luminosity profile decomposition shows that the model Gauss + bulge + disk properly reproduces the surface brightness of the galaxies. However, in order to account for the luminosity profile, most of the disk galaxies need the inner truncated exponential form with a central cutoff radius ranging from 3 to $10 \mathrm{kpc}$. This is interpreted in terms of reddened regions that are well identified in the $B-V$ color maps. These regions present very similar colors among them, with $(B-V) \sim 1.2$. This fact could be associated with the presence of dust confined in the inner regions of the galaxies.
\end{abstract}

Key words. galaxies: photometry - galaxies: active - galaxies: Seyfert

\section{Introduction}

The relationship between an active galactic nucleus (AGN) and its host galaxy is one of the key issues in the study of nuclear activity. One would expect that certain properties of active galaxies such as mass, luminosity, bulge to disk ratio and colors could influence nuclear activity or vice versa. In fact, the unified model for an AGN requires gas accretion, most probably from the host galaxy, onto a massive and compact object (Malkan 1983). Similarly, the nuclear burst scenario (Terlevich et al. 1992) requires gas fueling together with an efficient way to concentrate that gas into a small region of space, in a relatively short time-scale. Besides this, gas fueling seems to be connected to the presence of a bar, which is needed to get the non-axisymmetric potential that arises in from the theoretical works (Barnes \& Hernquist 1991). However,

\footnotetext{
Send offprint requests to: C. J. Donzelli,

e-mail: charly@oac.uncor.edu

* Visiting Astronomer, CTIO, NOAO, which is operated by AURA Inc., under contract to the NSF.

** On leave of absence from Observatorio Astronómico Nacional, Universidad Nacional de Colombia, Bogotá.
}

recent observational studies based on optical data show that barred galaxies are not a specific signature of nuclear activity (Ho et al. 1997; Hunt et al. 1999). In this sense, Regan \& Mulchaey (1999), based on the analysis of 12 Seyferts galaxies imaged with the HST, proposed central spiral dust lanes as an alternative mechanism to drive the gas to the central regions.

Another important question to consider is the role played by the environment as a trigger of nuclear activity. De Robertis et al. (1998) have found that AGNs are not more likely to be associated with interactions than normal galaxies. However, Pastoriza et al. (1999), studying a sample of interacting galaxies, found that almost $40 \%$ of the galaxies may host a low luminosity AGN.

It is also worth noting that most of the studies on the topics mentioned above are focused on the galaxy nuclei and some of them on the circumnuclear regions, but only a few works have paid attention to the properties of the stellar populations of the hosts galaxies. As an example, González-Delgado et al. (1997)(hereafter GD97), presented $\mathrm{H} \alpha$ images of a sample of 55 active galaxies and Sánchez-Portal et al. (2000) gave results of broad band $V R I$ and narrow band $\mathrm{H} \alpha$ photometry for a sample 
of 24 nearby active galaxies. Also, Hunt et al. (1997) and Márquez et al. (2000) presented near infrared broad band images of a sample of 26 and 18 active galaxies respectively.

Our goal in this paper is to present a new valuable set of photometric data for a sample of 10 Seyfert 1 galaxies and to describe the main properties of both the stellar component and the gas of the hosts galaxies. The paper is organized as follows: In Sect. 2 we describe the sample selection. In Sect. 3 we summarize the observations and data reduction. Section 4 discusses the photometric results and Sect. 5 describes the particular properties of each galaxy of the sample. Finally, in Sect. 6 we provide our conclusions.

\section{The sample}

The galaxies chosen for this study were selected to span as broad a range of nuclear magnitudes and optical line characteristics as possible and still be classified as Seyfert 1 or Narrow Line Seyfert 1 as is the case for $1 \mathrm{H} 1934$ 063. For example, the Full-width half maximum of the $\mathrm{H} \alpha$ line derived from spectroscopy varies from $2000 \mathrm{~km} \mathrm{~s}^{-1}$ to $7000 \mathrm{~km} \mathrm{~s}^{-1}$ and the contribution of the stellar population to the observed continuum varies from negligible up to $80 \%$ (Rodriguez-Ardila et al. 2000, hereafter RPD2000). These galaxies were selected from the compilation of many catalogs, mainly from the Calan Tololo Survey (Maza et al. 1989, 1992, 1994) and from the compilation of Véron-Cetty \& Véron (1996). However, due to limitations of the available observing time and the location of the observatory, the exact sample was a randomly selected subsample of our entire list of Seyfert 1. Those with little or no photometric information available in the literature were chosen for this publication.

Figures $1 \mathrm{a}-\mathrm{j}$ present our $V$ images of the sample galaxies. We have observed them to obtain photometric parameters such as isophotal shapes, colors and luminosity profiles that can provide information on their basic properties and structure. As a secondary objective, we want to contribute to the available multicolor photometric data for Seyfert 1 galaxies, that is still far from being completed. The continuum and line emission properties of six objects of our sample (CTS C16, CTS F10.01, CTS A08, 1H 2107097, CTS G03.04 and 1H 1934-063) were already studied by means of optical and near-IR spectroscopy (RPD2000).

The galaxies in our sample are listed in Table 1 along with information about their morphological type, magnitudes, extinction in blue band and radial velocities. These parameter were taken from the $\mathrm{NED}^{1}$ database. Throughout this paper we have adopted a Hubble constant $H_{0}=75 \mathrm{~km} \mathrm{~s}^{-1} \mathrm{Mpc}^{-1}$.

1 The NASA/IPAC Extragalactic Database (NED) is operated by the Jet Propulsion Laboratory, California Institute of Technology, under contract with the National Aeronautics and Space Administration.

\section{Observations and data reduction}

\subsection{Observations}

The sample was observed using the TEK 2048 CCD attached to the $0.9 \mathrm{~m}$ telescope of CTIO over 3 nights in August 1998. The pixel scale, projected to the sky plane, is $0.396^{\prime \prime}$. Broad $B, V, I$ and four narrow filters $75 \AA$ wide centered at $\lambda=6477 \AA, 6649 \AA, 6781 \AA$ and $7053 \AA$ were employed. The first narrow filter was used to measure the continuum level adjacent to $\mathrm{H} \alpha$ and the remaining three to isolate the $\mathrm{H} \alpha+\mathrm{N}[\mathrm{II}]$ emission, depending on the redshift of the object. Seeing conditions were very good $\left(1.2^{\prime \prime}\right.$ to $\left.1.5^{\prime \prime}\right)$ and all nights were photometric. For each target object we obtained a set (usually 3 or 5 ) of short (200 s$400 \mathrm{~s}$ ) exposures for each filter. A summary of the log of observations is shown in Table 2.

\subsection{Reduction of the $B V I$ and $H_{\alpha}$ images}

The images were corrected for bias and flat-field using standard IRAF routines ${ }^{2}$. Sky subtraction was performed by averaging mean values of the sky, well beyond the galaxy limits, in several boxes on each frame. All images for a particular galaxy were aligned and combined to obtain single $B, V, I$ and $\mathrm{H} \alpha$ images. The alignment was performed using the IMALIGN task of IRAF with at least 7 field stars. The typical accuracy was better than 0.05 pixels. The same technique was used to generate the $B-V$ and $B-I$ color images as well as to subtract the continuum emission from the $\mathrm{H} \alpha$ images. When combining frames, if the individual images had different seeing, the ones with better seeing were convolved with a Gaussian function in order to match the image with the poorer seeing.

The photometric calibration to the Johnson-Cousins system was made using 4 or 5 standard stars per night selected from Landolt (1992). The usual equations to transform the $b, v$ and $i$ instrumental magnitudes into the $V$, $(B-V)$ and $(V-I)$ standard magnitudes were used. Estimates of accuracy in the calibrations are $\pm 0.04 \mathrm{mag}$ in $V, \pm 0.06 \mathrm{mag}$ in $(B-V)$ and $\pm 0.06 \mathrm{mag}$ in $(V-I)$.

\section{Results}

\subsection{Integrated photometric parameters}

The morphological classification, based on the galaxy colors, and the calculated magnitudes and colors for the objects in the sample are listed in Table 3 . The magnitudes were derived by two independent methods. The first one is the integration of the intensity pixels in a series of diaphragms with increasing radius until the sum converges. The second method consisted of the integration of the luminosity profile (see Sect. 4.2). The results following the two methods are in very good agreement. However, the comparison with the magnitudes listed in The Third

\footnotetext{
${ }^{2}$ IRAF is distributed by NOAO, which is operated by AURA Inc., under contract to the NSF.
} 

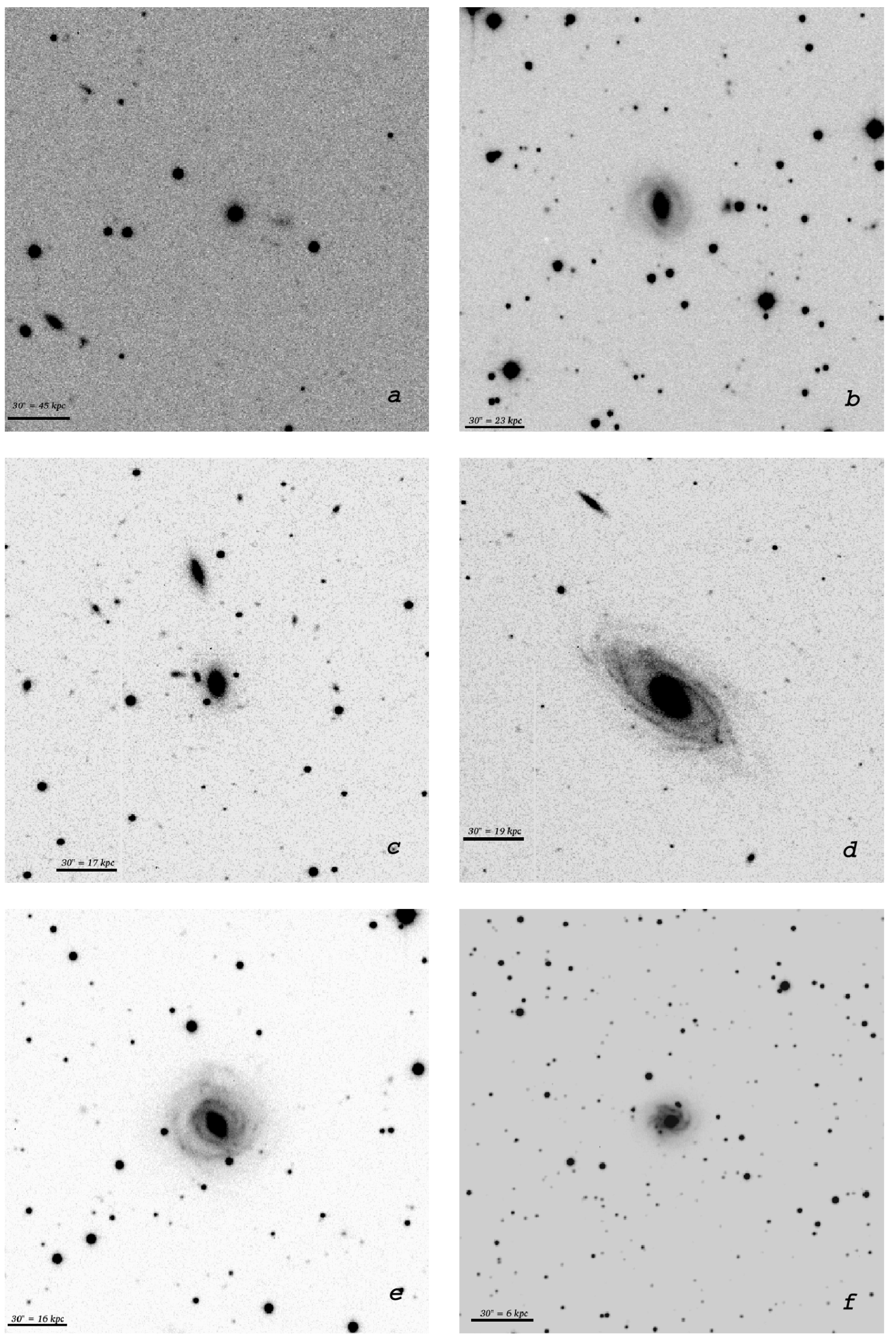

Fig. 1. V images of the sample galaxies. North is on top and East to the left. The lower left bar represents $30^{\prime \prime}$ and the corresponding projected scale in Kpc is also indicated. a) CTC C16.16, b) CTS G03.04, c) CTS A08.12, d) ESO 602- G031, e) ESO 025- G 002, f) 1H 1934-063, g) 1H 2107-097, h) ESO 354- G 004, i) MRK 0509, j) CTS F10.01. 

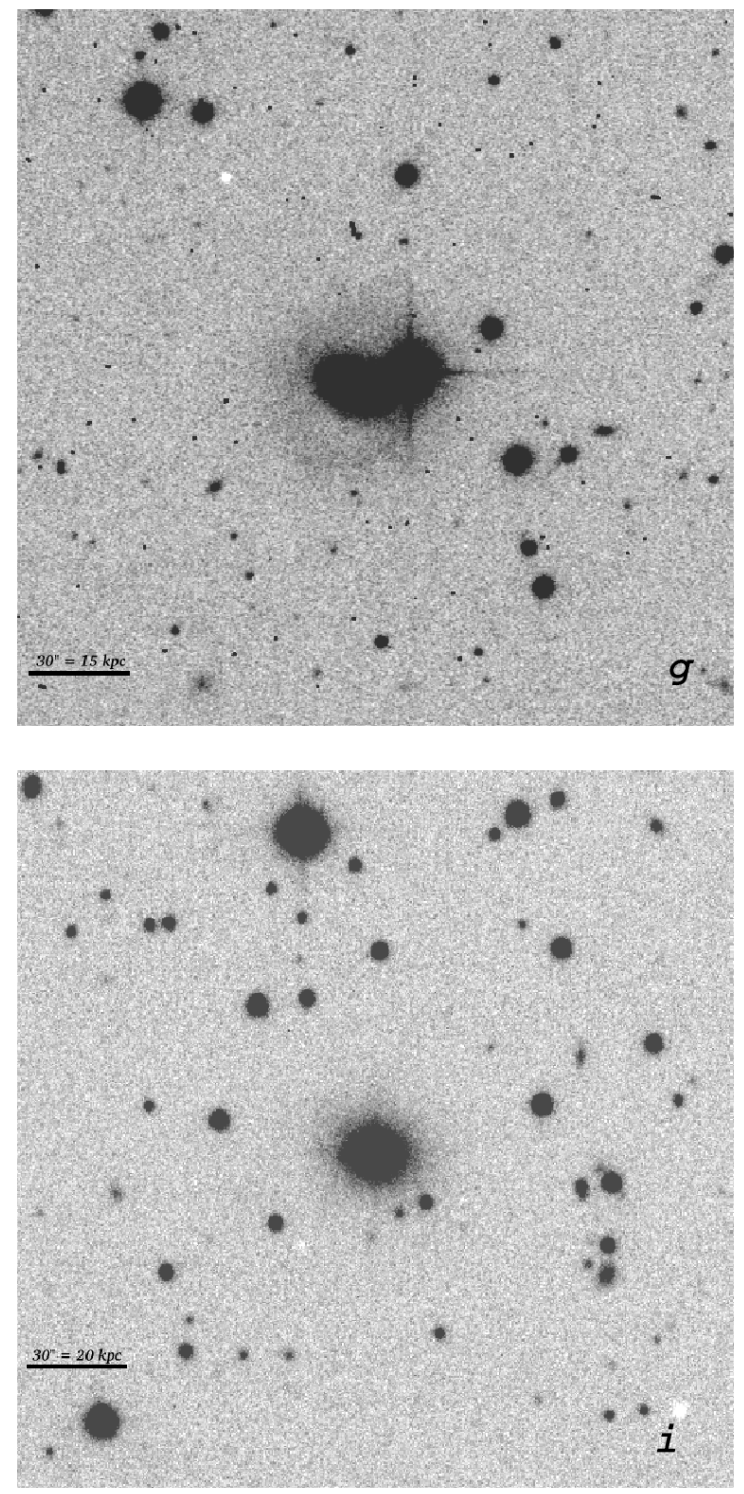
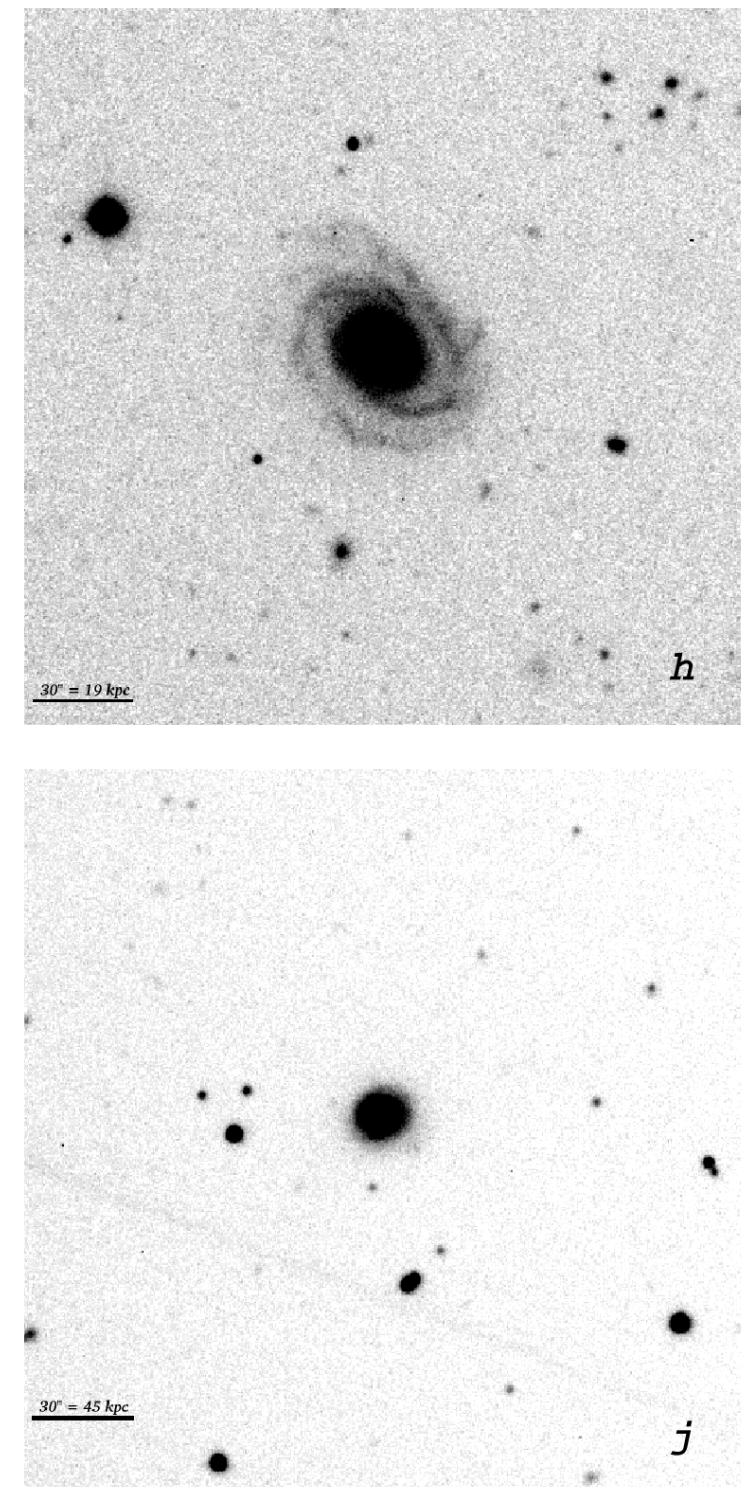

Fig. 1. continued.

Table 1. Basic information of the galaxy sample.

\begin{tabular}{lcccccc}
\hline & $\mathrm{RA}$ & $\alpha$ & & $\mathrm{cz}$ & & \\
Galaxy & $(\mathrm{J} 2000)$ & $(\mathrm{J} 2000)$ & $m_{\mathrm{b}}$ & $\left(\mathrm{km} \mathrm{s}^{-1}\right)$ & Morphology & $A_{\mathrm{b}}$ \\
\hline CTS C16.16 & 000053.5 & -443934 & 18.0 & 23825 & $\ldots$ & 0.06 \\
CTS G03.04 & 193804.5 & -510947 & 15.2 & 12082 & $(R) S B 0 / a$ & 0.27 \\
CTS A08.12 & 213202.2 & -334254 & 15.7 & 8780 & $\ldots$ & 0.23 \\
ESO 602-G 031 & 223655.9 & -221315 & 14.9 & 10101 & $\left(R^{\prime}\right) S A B(r s) b$ & 0.14 \\
ESO 025-G 002 & 185439.8 & -785351 & 14.6 & 8540 & $(R:) S A B(r) b$ & 0.69 \\
1H 1934-063 & 193733.2 & -061305 & 14.1 & 3174 & $E$ & 1.26 \\
1H 2107-097 & 210909.8 & -094016 & 14.3 & 8034 & $\ldots$ & 1.00 \\
ESO 354-G 004 & 015141.7 & -361116 & 15.1 & 10033 & $\left(R^{\prime}:\right)$ SA $(r s) b$ & 0.07 \\
MRK 509 & 204409.7 & -104325 & 13.0 & 10312 & compact & 0.25 \\
CTS F10.01 & 225551.9 & -294416 & 15.6 & 23500 & $\ldots$ & 0.09 \\
\hline
\end{tabular}

Reference Catalog of Bright Galaxies (de Vaucouleurs et al. 1991; hereafter RC3), are in a reasonable agreement only for ESO 602-G 031, ESO 025-G002, 1H 1934063, 1H 2107-097, and CTS F10.01. The remaining objects show differences up to $0.5 \mathrm{mag}$. This is the case for MRK 509 for which the RC3 lists $m_{\mathrm{b}}=13.0$ while our value is $m_{\mathrm{b}}=13.50$. Moreover, Kotilainen \& Ward (1994; hereafter KW94) obtained for the same galaxy $m_{\mathrm{b}}=14.06$. We attribute the discrepancies to variability of the AGN. 
Table 2. Log. of observations and exposure time in each filter ${ }^{\mathrm{a}}$.

\begin{tabular}{|c|c|c|c|c|c|c|c|c|}
\hline \multirow[b]{2}{*}{ Galaxy } & \multirow[b]{2}{*}{ Date } & \multirow[b]{2}{*}{$B$} & \multirow[b]{2}{*}{$V$} & \multirow[b]{2}{*}{$I$} & \multicolumn{4}{|c|}{$\mathrm{H} \alpha$} \\
\hline & & & & & $(\lambda 6477)$ & $(\lambda 6649)$ & $(\lambda 6781)$ & $(\lambda 7053)$ \\
\hline CTS C16.16 & 15 Aug. 98 & $5 \times 200$ & $5 \times 150$ & $5 \times 150$ & $\ldots$ & $\ldots$ & $\ldots$ & ... \\
\hline CTS G03.04 & 17 Aug. 98 & $3 \times 300$ & $3 \times 250$ & $3 \times 250$ & $\ldots$ & $\ldots$ & $\ldots$ & ... \\
\hline CTS A08.12 & 15 Aug. 98 & $3 \times 450$ & $3 \times 300$ & $3 \times 300$ & $3 \times 300$ & $\ldots$ & $3 \times 300$ & $\ldots$ \\
\hline ESO 602 - G 031 & 15 Aug. 98 & $3 \times 450$ & $3 \times 350$ & $3 \times 300$ & $3 \times 300$ & $\ldots$ & $3 \times 300$ & $\ldots$ \\
\hline ESO 025 - G 002 & 15 Aug. 98 & $3 \times 200$ & $3 \times 150$ & $3 \times 150$ & $4 \times 150$ & $\ldots$ & $4 \times 150$ & $\ldots$ \\
\hline 1H $1934-063$ & 15 Aug. 98 & $3 \times 400$ & $3 \times 250$ & $\ldots$ & $3 \times 250$ & $3 \times 250$ & $\ldots$ & $\ldots$ \\
\hline 1H $2107-097$ & 17 Aug. 98 & $3 \times 350$ & $3 \times 300$ & $3 \times 300$ & $\ldots$ & $\ldots$ & $\ldots$ & $\cdots$ \\
\hline ESO $354-$ G 004 & 16 Aug. 98 & $4 \times 400$ & $3 \times 300$ & $3 \times 300$ & $\cdots$ & $\ldots$ & $\ldots$ & $\ldots$ \\
\hline MRK 509 & 16 Aug. 98 & $3 \times 300$ & $3 \times 200$ & $3 \times 200$ & $3 \times 250$ & $\ldots$ & $3 \times 250$ & $\ldots$ \\
\hline CTS F10.01 & 16 Aug. 98 & $3 \times 400$ & $3 \times 300$ & $3 \times 300$ & $3 \times 350$ & $\ldots$ & $\ldots$ & $3 \times 350$ \\
\hline
\end{tabular}

${ }^{\text {a }}$ For each filter we list the total number of frames multiplied by the exposure time of each frame, in seconds.

Table 3. Morphologies, magnitudes and colors derived for the galaxy sample.

\begin{tabular}{lcccccc}
\hline Galaxy & Morphology $^{\mathrm{a}}$ & $D \times d^{\mathrm{b}}$ & $M_{\mathrm{b}}$ & $B$ & $(B-V)$ & $(V-I)$ \\
\hline CTS C16.16 & $E 1$ & $5.5 \times 5.5$ & -20.36 & 17.32 & 0.91 & 0.79 \\
CTS G03.04 & $S O$ & $17.4 \times 17.2$ & -20.70 & 15.46 & 0.80 & 0.88 \\
CTS A08.12 & $S O$ & $9.5 \times 9.2$ & -19.24 & 16.23 & 1.01 & 0.94 \\
ESO 602-G 031 & $S B a$ & $40.8 \times 34.1$ & -21.28 & 14.76 & 0.71 & 0.98 \\
ESO 025-G 002 & $S B a$ & $26.1 \times 26.1$ & -20.85 & 14.67 & 0.85 & 1.36 \\
1H 1934-063 & $S b$ & $19.8 \times 19.4$ & -18.74 & 14.20 & 0.61 & $\ldots$ \\
1H 2107-097 & $S O$ & $11.1 \times 10.2$ & -20.68 & 14.58 & 0.75 & 1.26 \\
ESO 354-G 004 & $S a$ & $34.4 \times 34.1$ & -21.22 & 14.65 & 0.88 & 1.13 \\
MRK 509 & $S O ?$ & $19.0 \times 18.8$ & -22.34 & 13.50 & 0.15 & 0.80 \\
CTS F10.01 & $S O$ & $12.3 \times 12.2$ & -21.99 & 15.60 & 0.67 & 0.86 \\
\hline
\end{tabular}

a This paper.

b Major and minor diameters in arcsec integrated up to $m_{\mathrm{b}}=25 \mathrm{mag} \operatorname{arcsec}^{-2}$.

The luminosity for the objects in the sample ranges from -18.7 to -22.3 with an average $M_{\mathrm{b}}=-20.7$. This value is rather similar to that found by Yee (1983), $M_{\mathrm{b}}=-20.4$, for a sample of Seyfert galaxies, but somewhat higher when compared to $M_{\mathrm{b}}=-20.0$ found by KW94 for a sample of Seyfert 1 galaxies. On the other hand, Christensen (1975) found that for a sample of normal spiral galaxies $M_{\mathrm{B}}=-19.7$. Note that $M_{\mathrm{b}}$ values given by the other authors have been recalculated using $H_{0}=75 \mathrm{~km} \mathrm{~s}^{-1} \mathrm{Mpc}^{-1}$.

\subsection{Luminosity profiles}

Since our sample is composed of both elliptical and spiral galaxies, we have used two different methods in order to obtain surface brightness profiles. For elliptical galaxies, profiles were obtained using the ELLIPSE routine within STSDAS (Jedrezejewski 1987). Basically the task starts from a first guess elliptical isophote defined by approximate values for the center coordinates, ellipticity and position angle. With these initial values the image is sampled along an elliptical path producing an intensity distribution as a function of the position angle. Then the harmonic content of this distribution is analyzed by leastsquares. The harmonic amplitudes, together with the local image radial gradient, are related to a specific ellipse geometric parameter and give information on how much the current parameter value deviates from the true one. The parameter is then modified by the calculated value and the process continues until convergence is reached.

The approach was different for spiral galaxies because the ELLIPSE algorithm does not converge due to the clumpy structure present in the spiral arms. In this case we have used the equivalent profile $m$ versus $r_{\text {eq }}$, where $r_{\text {eq }}=(S / \pi)^{1 / 2}$ being $S(m)$ proportional to the area projected on the image (in square arcsecs) subtended by all those points of the galaxy for which the intensity $I\left(m^{\prime}\right)=10^{-0.4 m}$ satisfies the condition $I\left(m^{\prime}\right)>I(m)$ 
(Sérsic 1982). It is worth noting that this last profile and that used for an elliptical galaxy show exactly the same behavior for an E0 galaxy.

The $B, V$ and $I$ profiles were then decomposed into 3 assumed components: Gaussian (due to the stellar-like profile of the AGN), bulge and disk. In three cases we noted the presence of other components such as bars, arms or lens. These structures have not been taken into account in the fit because their contribution to the total luminosity of the galaxy were not greater than $5 \%$. The functional form adopted for each of the fitting component is as follows:

$I(r)=I_{0} \exp \left(-2.71 *(r / f w)^{2}\right)$

for the Gaussian;

$I(r)=I_{\mathrm{e}} \exp \left(-7.688 *\left(\left(r / r_{\mathrm{eff}}\right)^{.25}-1\right)\right)$

for the bulge;

$I(r)=I_{\mathrm{d}} \exp \left(-r / d_{\mathrm{l}}-\left(h_{\mathrm{d}} / r\right)^{3}\right)$

for the disk component.

In the above expressions $I_{0}$ is the peak of the Gaussian profile at $r=0$ and $f w$ the full width at half maximum (FWHM). The quantity $I_{\mathrm{e}}$ is the intensity at $r_{\text {eff }}$, the radius that encloses half of the total luminosity of the bulge (also known as the effective radius). Finally, $I_{\mathrm{d}}$ is the central intensity, $d_{\mathrm{l}}$ the length scale and $h_{\mathrm{d}}$ the radius of central cutoff of the disk component.

In order to obtain the above parameters we followed the method described by Shombert \& Bothum (1987) using the NFIT routine implemented in STSDAS. This routine must be provided with appropriate initial parameters in order to begin the fit. Disk parameters can be guessed directly through the profile since the disk is not seriously contaminated by the bulge in the outermost region of the galaxy profile. However, it is necessary to have photometric data at large radii in order to avoid contamination from other more central components (Prieto et al. 1992). The main difficulty was to perform the fit to the Gaussian and bulge components since they completely overlap each other. This problem was solved by performing an initial fit considering only the innermost region data, generally the first $4-5^{\prime \prime}$. During this process we fixed the disk guessed parameters and the $f w$ value that was initially calculated using field stars. Task converges rapidly at this step and calculated parameters do not significantly depend on the initial adopted values. Finally, the calculated parameters for the Gaussian and bulge components together with those of the disk were used as initial values to perform the fit over the whole range of the luminosity profile, omitting those points affected by additional structures. Uncertainties in the parameters were calculated by doing small variations on the initial adopted values prior to the fitting. We found that the differences were never greater than $20 \%$. We have also checked how seeing influences the calculated parameters by deconvolving the images. The most seriously affected parameters are those of the bulge
( $I_{0}$ tends to be higher and $r_{\mathrm{e}}$ tends to be smaller) but the variations were never greater than 10-15\%. Moreover, as deconvolution is a conservative process, the luminosity ratios within the components remain unchanged between the errors.

From the derived photometric parameters we were then able to calculate the total luminosity for each of the 3 components by integrating Eqs. (1)-(3) as follows:

$L=\int_{0}^{\infty} I(r) 2 \pi r \mathrm{~d} r$

This integration leads to the following results:

$L_{\mathrm{AGN}}=\frac{\pi I_{g 0} f w^{2}}{2.71}$

for the Gaussian

$L_{\text {bulge }}=7.21 \pi I_{\mathrm{e}} r_{\mathrm{eff}}^{2}$

for the bulge

$L_{\text {disk }}=2 \pi I_{0} d_{1}^{2}$

for the disk component when $h_{\mathrm{d}}=0$.

For the case $h_{\mathrm{d}} \neq 0$, the above integral has no analytic solution, so a numerical integration was adopted. Total magnitudes obtained using the above procedure are, on average, $0.10 \mathrm{mag}$ brighter than those calculated through the integration of the image intensity pixels, as was explained in Sect. 4.1. This systematic difference is not surprising since now the luminosity profile integration is made up to $r=\infty$.

Figures $2 \mathrm{a}-\mathrm{j}$ present the observed luminosity profiles and the fit applied to each galaxy. Color profiles have been obtained directly from the fitted profiles. Decomposition in the three components is only shown for the $B$ data. Table 4 lists the photometric parameters calculated from the decomposition technique and Table 5 lists the bulge to disk, bulge to Gaussian and Gaussian to total luminosity ratios in the $B, V$ and $I$ filters. Note that in Table $4, g_{0}=$ $-2.5 \log \left(I_{0}\right), m_{\mathrm{e}}=-2.5 \log \left(I_{\mathrm{e}}\right)$ and $b_{0}=-2.5 \log \left(I_{\mathrm{d}}\right)$. These quantities are expressed in mag $\operatorname{arcsec}^{-2}$.

A very interesting result was obtained from the decomposition profiles: In six out of eight disk galaxies it was necesary to truncate the exponential profile in order to get an adecuate fit. In fact, the radius of the central cutoff $h_{\mathrm{d}}$ ranged from $3 \mathrm{kpc}$ for CTS A08.12 and 1H 2107-097, up to $10 \mathrm{kpc}$ as is the case of ESO 602-G031. Moreover, for each galaxy the cutoff radius corresponds to a reddened region that is well identified in the $B-V$ color map. We have obtained very similar colors for these regions, $(B-V) \sim 1.2$. Color maps for three galaxies of the sample are presented in Sect. 5.

\subsection{Color diagram and gradients}

The dominant stellar population of the individual galaxies is inferred from the integrated colors. This information, 
Table 4. Photometric parameters derived for the galaxy sample.

\begin{tabular}{|c|c|c|c|c|c|c|c|c|c|c|c|c|c|c|c|c|c|c|c|c|c|}
\hline \multirow[b]{2}{*}{ Galaxy } & \multicolumn{3}{|c|}{$g_{\mathrm{o}}^{\mathrm{a}}$} & \multicolumn{3}{|c|}{$f_{\mathrm{w}}^{\mathrm{b}}$} & \multicolumn{3}{|c|}{$m_{\mathrm{e}}^{\mathrm{a}}$} & \multicolumn{3}{|c|}{$r_{\mathrm{e}}^{\mathrm{c}}$} & \multicolumn{3}{|c|}{$b_{\mathrm{o}}^{\mathrm{a}}$} & \multicolumn{3}{|c|}{$d_{1}^{\mathrm{c}}$} & \multicolumn{3}{|c|}{$h_{\mathrm{d}}^{\mathrm{c}}$} \\
\hline & $B$ & $V$ & $I$ & $B$ & $V$ & $I$ & $B$ & $V$ & $I$ & $B$ & $V$ & $I$ & $B$ & $V$ & $I$ & $B$ & $V$ & $I$ & $B$ & $V$ & $I$ \\
\hline CTS C16.16 & 20.1 & 19.8 & 19.2 & 2.0 & 2.2 & 1.8 & 21.3 & 20.3 & 19.5 & 2.0 & 3.1 & 2.2 & $\ldots$ & .. & $\cdots$ & $\ldots$ & $\ldots$ &. & $\ldots$ & .. & $\cdots$ \\
\hline CTS G03.04 & 18.3 & 17.6 & 16.7 & 1.5 & 1.5 & 1.3 & 20.9 & 20.4 & 21.0 & 1.2 & 1.6 & 3.7 & 22.8 & 21.7 & 21.0 & 6.8 & 5.6 & 5.2 & .. & .. & $\ldots$ \\
\hline CTS A08.12 & 18.2 & 17.5 & 17.7 & 1.6 & 1.4 & 1.3 & 20.8 & 19.7 & 18.4 & 0.7 & 0.8 & 0.6 & 24.5 & 23.9 & 22.4 & 5.5 & 7.3 & 7.7 & 3.4 & 2.4 & 1.4 \\
\hline ESO $602-$ G 031 & 19.2 & 18.7 & 18.8 & 1.6 & 1.8 & 1.7 & 24.3 & 23.3 & 22.1 & 11.5 & 9.8 & 8.7 & 22.0 & 21.7 & 20.0 & 6.2 & 6.2 & 6.2 & 10.2 & 10.5 & 12.0 \\
\hline ESO 025 - G 002 & 20.1 & 19.7 & 18.6 & 2.1 & 2.1 & 2.0 & 23.0 & 22.2 & 20.7 & 3.7 & 4.4 & 4.2 & 21.9 & 20.8 & 19.8 & 6.1 & 5.0 & 5.2 & 3.9 & 4.6 & 4.7 \\
\hline 1H $1934-063$ & 17.8 & 18.1 & $\ldots$ & 2.0 & 2.0 & $\ldots$ & 24.3 & 20.7 & $\ldots$ & 2.3 & 0.5 & $\ldots$ & 20.4 & 20.1 & $\ldots$ & 1.0 & 1.2 & $\ldots$ & $\ldots$ & $\ldots$ & $\ldots$ \\
\hline $1 \mathrm{H} 2107-097$ & 17.2 & 17.2 & 16.3 & 1.9 & 1.8 & 1.7 & 18.7 & 17.9 & 19.0 & 0.6 & 0.6 & 1.6 & 20.3 & 20.0 & 19.3 & 1.8 & 2.3 & 3.3 & 3.4 & 2.8 & 2.9 \\
\hline ESO $354-$ G 004 & 19.8 & 18.8 & 17.6 & 2.3 & 2.0 & 2.8 & 21.7 & 20.6 & 20.4 & 3.3 & 3.2 & 4.9 & 22.1 & 21.9 & 20.1 & 6.6 & 6.6 & 4.4 & 10.3 & 10.0 & 9.7 \\
\hline MRK 509 & 16.5 & 16.7 & 16.6 & 2.9 & 2.8 & 2.8 & 18.3 & 18.2 & 17.1 & 0.9 & 1.1 & 1.0 & 21.7 & 20.8 & 19.6 & 3.5 & 3.5 & 3.5 & 8.7 & 8.7 & 8.7 \\
\hline CTS F10.01 & 19.1 & 18.4 & 17.9 & 2.4 & 2.1 & 2.1 & 19.7 & 19.3 & 18.2 & 1.2 & 1.4 & 0.9 & 20.7 & 20.1 & 19.0 & 4.6 & 4.9 & 5.0 & ... & $\ldots$ & $\cdots$ \\
\hline
\end{tabular}

${ }^{\mathrm{a}}$ In units of mag $\operatorname{arcsec}^{-2}$.

b In units of arcseconds (").

c In units of kpc.

Table 5. Luminosity ratios between components.

\begin{tabular}{|c|c|c|c|c|c|c|c|c|c|}
\hline \multirow[b]{2}{*}{ Galaxy } & \multicolumn{3}{|c|}{$L_{\text {bulge }} / L_{\text {disk }}$} & \multicolumn{3}{|c|}{$L_{\text {bulge }} / L_{\text {Gauss }}$} & \multicolumn{3}{|c|}{$L_{\text {Gauss }} / L_{\text {total }}$} \\
\hline & $B$ & $V$ & $I$ & $B$ & $V$ & $I$ & $B$ & $V$ & $I$ \\
\hline CTS C16.16 & $\ldots$ & $\ldots$ & $\ldots$ & 2.7 & 4.3 & 8.9 & 0.27 & 0.19 & 0.10 \\
\hline CTS G03.04 & 0.6 & 1.0 & 1.8 & 2.0 & 2.9 & 5.6 & 0.16 & 0.15 & 0.10 \\
\hline CTS A08.12 & 2.0 & 2.0 & 1.0 & 1.2 & 2.5 & 7.6 & 0.36 & 0.21 & 0.06 \\
\hline ESO $602-$ G 031 & 3.9 & 6.1 & 3.3 & 43.0 & 20.7 & 56.0 & 0.02 & 0.04 & 0.01 \\
\hline ESO 025 - G 002 & 0.6 & 1.1 & 1.5 & 13.5 & 27.2 & 41.5 & 0.03 & 0.02 & 0.01 \\
\hline 1H $1934-063$ & 0.6 & 0.4 & ... & 1.6 & 3.2 & $\ldots$ & 0.18 & 0.09 & ... \\
\hline 1H $2107-097$ & 4.2 & 3.2 & 1.7 & 1.7 & 4.4 & 5.5 & 0.33 & 0.15 & 0.10 \\
\hline ESO $354-$ G 004 & 3.0 & 6.2 & 11.9 & 16.8 & 22.5 & 10.9 & 0.04 & 0.04 & 0.08 \\
\hline MRK 509 & 22.1 & 16.1 & 7.1 & 0.8 & 1.6 & 3.5 & 0.56 & 0.37 & 0.20 \\
\hline CTS F10.01 & 0.6 & 0.6 & 0.3 & 1.2 & 1.6 & 1.2 & 0.24 & 0.19 & 0.14 \\
\hline
\end{tabular}

together with the luminosity profiles, can be used to derive the morphological type of the host galaxies. Figure 3 illustrates the color-color diagram $(B-V)$ vs. $(V-I)$ for the galaxies listed in Table 3 . We have compared our data with those obtained from Hunt et al. (1999) for a sample of Seyfert 1 galaxies. The colors of both samples show a similar behavior. We have also plotted in that figure the integrated averaged colors of normal galaxies, taken from Table 2 of de Jong et al. (1996), for two different morphological types as well as Stellar population models for E galaxies with ages between 12 and 15 Gyr computed by Tantalo et al. (1998).

From the color-color diagram we conclude that: a) $40 \%$ of the sample (ESO 602-G 031, 1H 1934-063, 1H2107-097, and CTSF10.01) has a stellar population typical of Seyfert galaxies hosted by a spiral galaxy; b) CTS C16.16 and CTS A08.12 are early-type galaxies; c) The integrated $(B-V)$ color of MRK 509 is bluer than that expected for any morphological type. However, it should be stressed that nearly half the luminosity of MRK 509 comes from its AGN.
A quick inspection of the color profiles in Figs. $2 \mathrm{a}-\mathrm{j}$ shows that almost all galaxies present color gradients. In 1H 1934-063, 1H 2107-097, MRK 509 and CTS F10.01, their $B-V$ and $V-I$ colors become monotonically redder with increasing distance to the galaxy center. In addition, the Seyfert nucleus leaves a clear signature in the color profiles: in the central $3^{\prime \prime}$ colors are, on average, $0.3 \mathrm{mag}$ bluer than those of the external parts of the galaxy. However, ESO 602-G 031 and ESO 354-G 004 show redder $B-V$ color gradients towards the center but their $V-I$ color profiles are almost constant along the galaxy radius. It is worth noting that $1 \mathrm{H} 2107-097$ has a $V-I$ color profile abnormally red $(V-I \sim 1.8)$ at large radius.

Color profiles can be interpreted in terms of the spectral energy distribution of a given galaxy. Our sample is composed of nine Seyfert 1 galaxies and one NarrowLine Seyfert 1 galaxy. These type of galaxies have strong emission lines and a continuum which can be accounted for by a combination of stellar population and a nonthermal spectrum. A significant difference in line intensity ratios and in the optical spectral index is observed among 

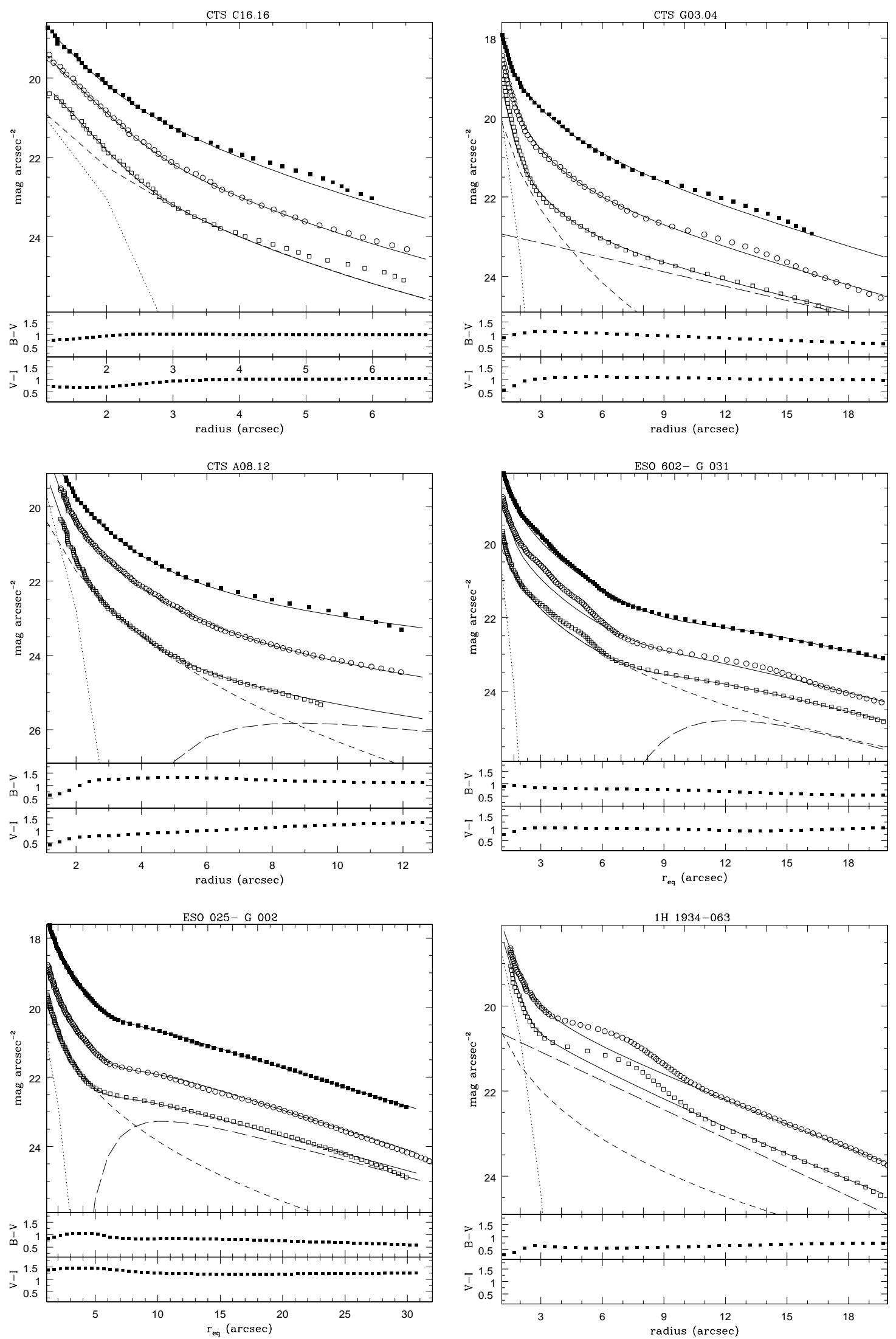

Fig. 2. Luminosity $B$ (empty squares), $V$ (empty circles), $I$ (filled squares) and color $B-V$ and $V-I$ profiles of the sample galaxies. Solid line is the best fit to the data. Decomposition in a Gauss (points) bulge (short dashed) and disk components (long dashed) is only shown for the $B$ data. Color $B-V$ and $V-I$ profiles were calculated using the obtained fitted functions. a) CTC C16.16, b) CTS G03.04, c) CTS A08.12, d) ESO 602 - G031, e) ESO 025 - G 002, f) 1H 1934-063, g) 1H 2107-097, h) ESO 354 - G 004, i) MRK 0509, j) CTS F10.01. 

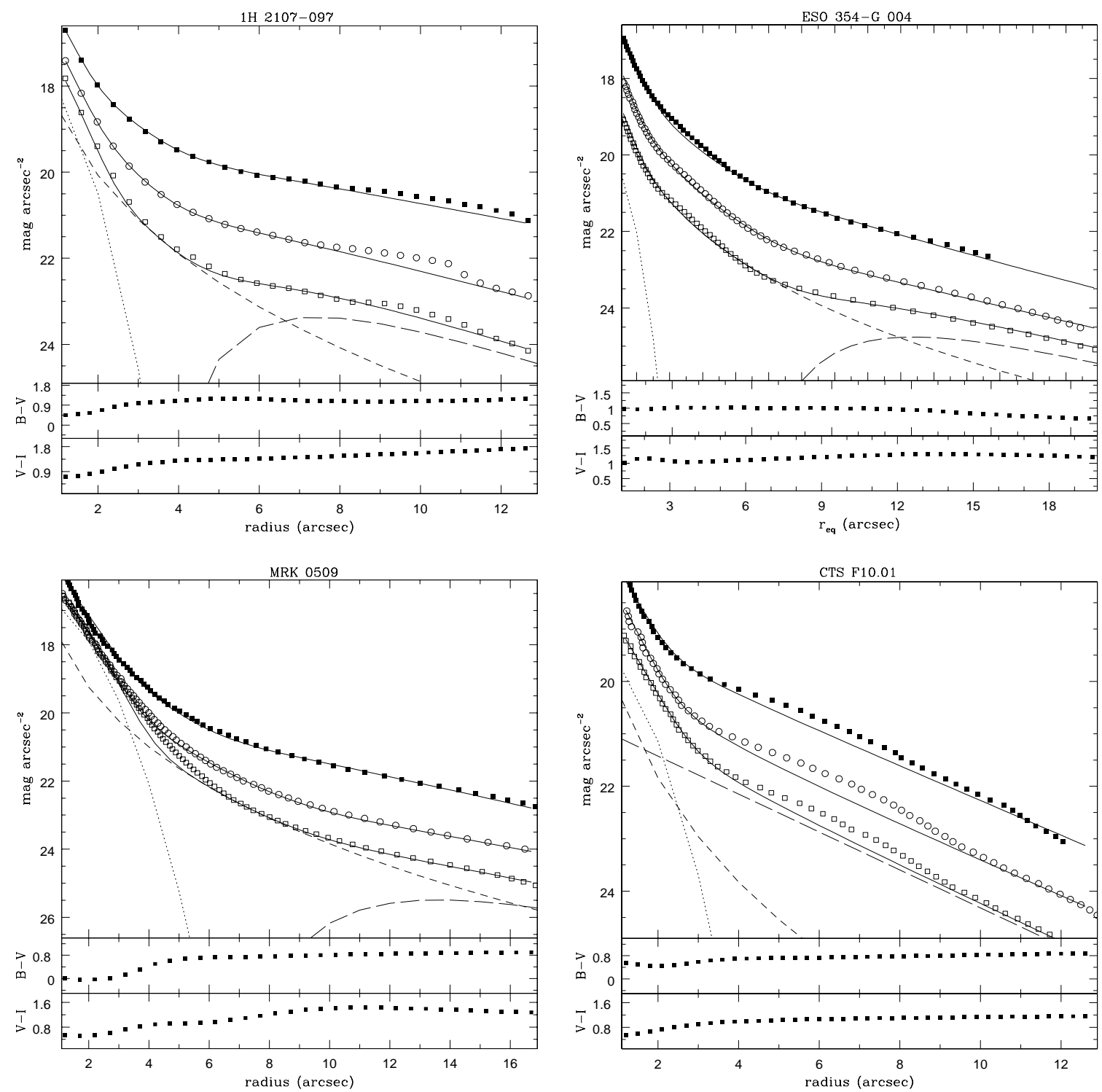

Fig. 2. continued.

Seyfert 1 and Narrow Line Seyfert 1 galaxies (RPD2000). Seyfert 1s, like CTS C16.16, A08.12, CTS F10.01 and CTS G03.04 have continua steeper to the blue (RPD2000), which is consistent with the observed color profiles. The galaxy 1H 2107-097, has a flat continumm (Fig. 1c in RPD2000) and it is the only galaxy in our sample that shows peculiar color profiles. The $B-V$ profile is very blue in the galaxy center while the $V-I$ is abnormally red.

\subsection{Ho images}

It is surprising to see that only one out of the $6(16 \%)$ imaged galaxies shows $\mathrm{H} \alpha$ emission beyond the nucleus. In fact, we detected disk emission in ESO 025-G 002, as can be seen from the continuum subtracted $\mathrm{H} \alpha+[\mathrm{NII}]$ image presented in Fig. 4. From this image we can appreciate that emission is extended up to a distance of $8 \mathrm{kpc}$ from the nucleus.

The previous result is different from that reported by Pogge (1989) (hereafter P89) who found that 3 out of 9 $(33 \%)$ of the Seyfert 1 galaxies showed extended emission within the inner $1 \mathrm{kpc}$. Moreover, P89 results contrast with those found by GD97 that report that 8 out of $13(61 \%)$ Seyfert 1 galaxies showed extended emission. However, we must note that the mean distance for our sample is $v z=10500 \mathrm{~km} \mathrm{~s}^{-1}$, leading to a scale length of $0.7 \mathrm{kpc} \operatorname{arcsec}^{-1}$. This is well below the resolution of both P89 and GD97 works, for which the mean distance of the samples is $v z \sim 2000 \mathrm{~km} \mathrm{~s}^{-1}$ that leads to a scale length of $0.1 \mathrm{kpc} \operatorname{arcsec}^{-1}$.

On the other hand, another important issue to have in mind is how these samples were defined. In principle, the P89 and GD97 samples are similar, but while GD97 do not use interaction as a selecting criterion, P89 excludes interacting galaxies. Similarly, P89 does not limit the sample by inclination angle while GD97 do. None of these selecting critera was used to define our sample. 


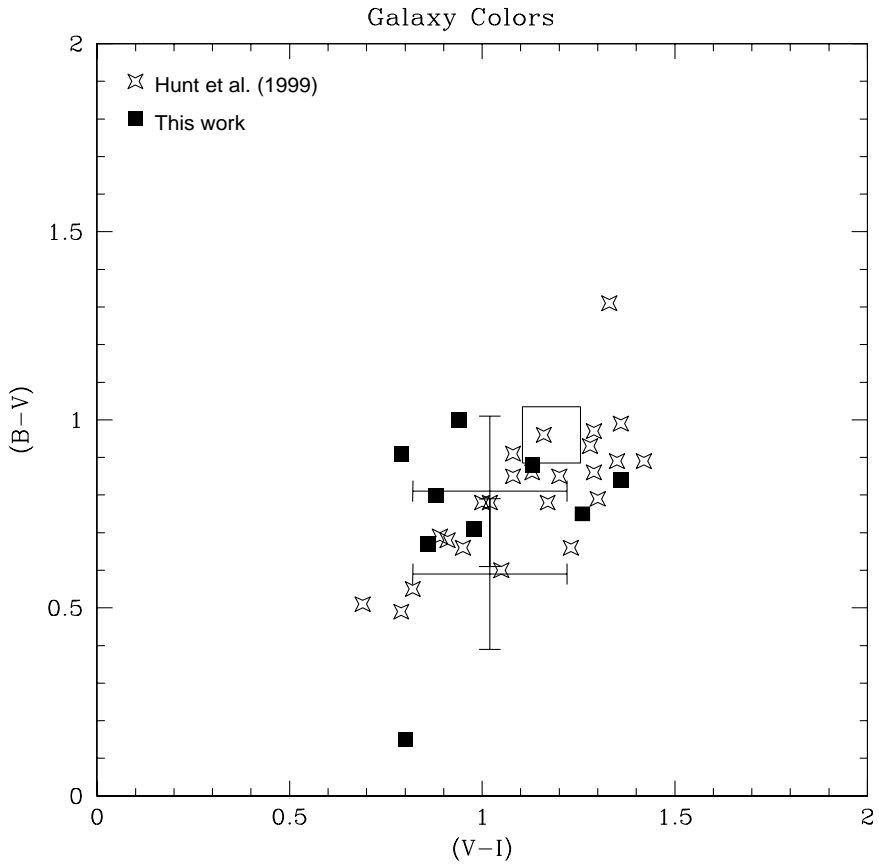

Fig. 3. Color-color diagram for the sample galaxies (filled squares). Stars represent the Hunt et al. (1999) sample. Upper and lower crosses show the average color for normal galaxies with $0<T<2$ and $6<T<8$ respectively. The small box show color models for elliptical galaxies with ages between 12 and 15 Gyr taken from Tantalo et al. (1998).

\section{The galaxies}

In this section we describe the main photometric properties of the galaxies of the sample.

CTS C16.16: It appears in our images as elliptical (see Fig. 1a). Its luminosity profile follows the $r^{1 / 4}$ up to $4^{\prime \prime}$. Beyond this limit the profile shows an excess which may be suggestive of a merger scenario. In fact, we observe from our images a group of three small galaxies with very disturbed isophotes towards the SW of CTS C16.16. The biggest one, located to the $\mathrm{W}$, is rather blue with $(B-V)=0.6$. Color profiles show that outward of $3^{\prime \prime}$ CTSC16.16 does not present a significant gradient, but within this radius both color profiles become bluer. We interpret this result as due to the luminosity of the Gaussian component, which contributes to $30 \%$ of the total luminosity. The integrated $(B-V)$ and $(V-I)$ colors are consistent with a dominant stellar population typical of an early type galaxy. From the absolute blue magnitude of CTS C16.16 $\left(M_{B}=-20.36\right)$ and its dimension $\left(r_{\text {eff }}=2 \mathrm{kpc}\right)$ we conclude that this object is a compact elliptical galaxy.

CTS G03.04: It is a ringed SO galaxy (see Fig. 1b). Besides the ring, shells and plumes are also observed in our images. Despite its morphological classification, CTS G03.04 has rather blue integrated colors $[(B-V)=0.80,(V-I)=$ $0.88)]$, quite similar to those of an early spiral (Sa). We suggest that such colors may mostly be due to the AGN

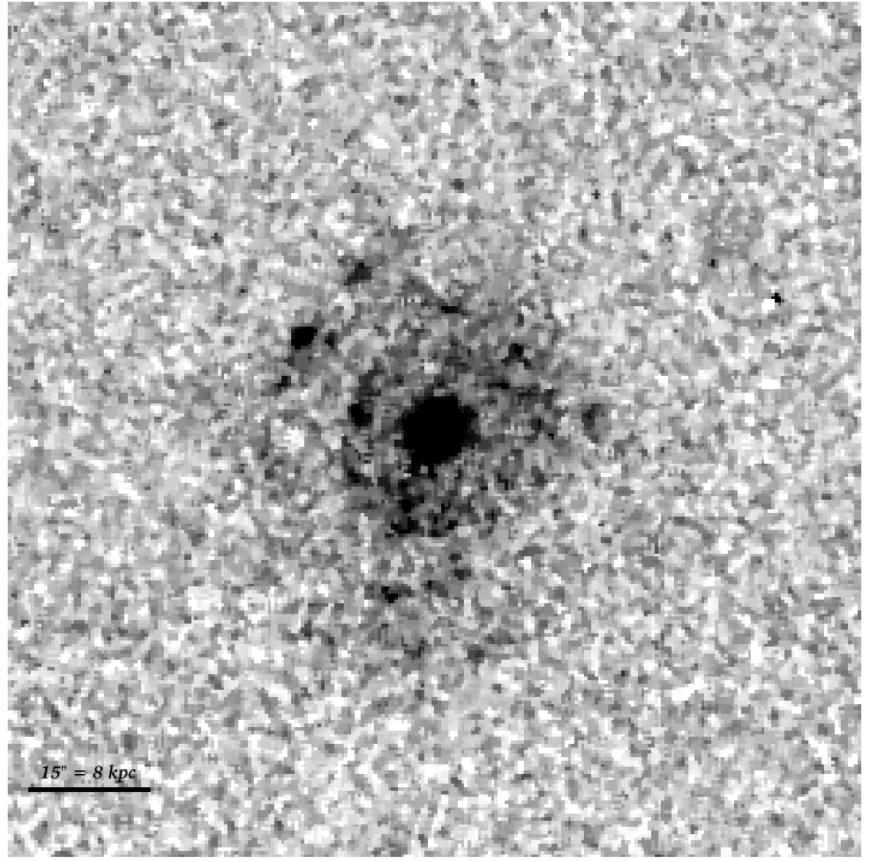

Fig. 4. $\mathrm{H} \alpha$ line emission image of ESO 025-G 032. North is on top and East to the left. The lower left bar represents $15^{\prime \prime}$ and the corresponding projected scale in $\mathrm{kpc}$ is also indicated.

and disk luminosities. In fact, the blue disk luminosity contribution is almost a factor of 2 larger than that of the bulge and the AGN contributes with almost $20 \%$ of the total galaxy luminosity. Color profiles show that the disk becomes important from $6^{\prime \prime}$ outwards. Inwards, the gradient becomes blue. The profiles also become very blue towards the galaxy center, where the Seyfert nucleus dominates. A small galaxy, with $(B-V)=0.81$, is located at $31^{\prime \prime}$ to the west of CTS G03.04.

CTS A08.12: This object appears in our images as an E2 (see Fig. 1c) but according to its luminosity profile, we classified it as SO due to the presence of a disk that accounts for $50 \%$ of the bulge luminosity. In order to obtain a satisfactory fit to the luminosity profile the disk needed a cutoff radius at about $3 \mathrm{kpc}$. The integrated colors are similar to a dominant stellar population of an E galaxy. The $B-V$ profile shows a blue gradient that begins at $r=6^{\prime \prime}$ outwards due to the presence of this disk. However, the $V-I$ profile shows a red gradient with a crescent radius. The most external isophotes of CTS A08.12 are rather disturbed, probably by the presence of three small galaxies located at $9^{\prime \prime}, 17^{\prime \prime}$ and $18^{\prime \prime}$ to the NE from the nucleus. The colors of these three objects are very similar, with $(B-V) \sim 1.7$. Towards the $N$, at about $50^{\prime \prime}$, there is another SO galaxy that shows very disturbed inner isophotes. A closer inspection of the whole frame field (about $0.09 \mathrm{Mpc}^{2}$ ) shows more than 20 galaxies brighter than $m_{B}=18.5$ and with $(B-V)$ colors ranging from 1.2 to 1.8. This evidence strongly suggests that CTS A08.12 is located in a group or a poor cluster, not yet identified in the literature. 

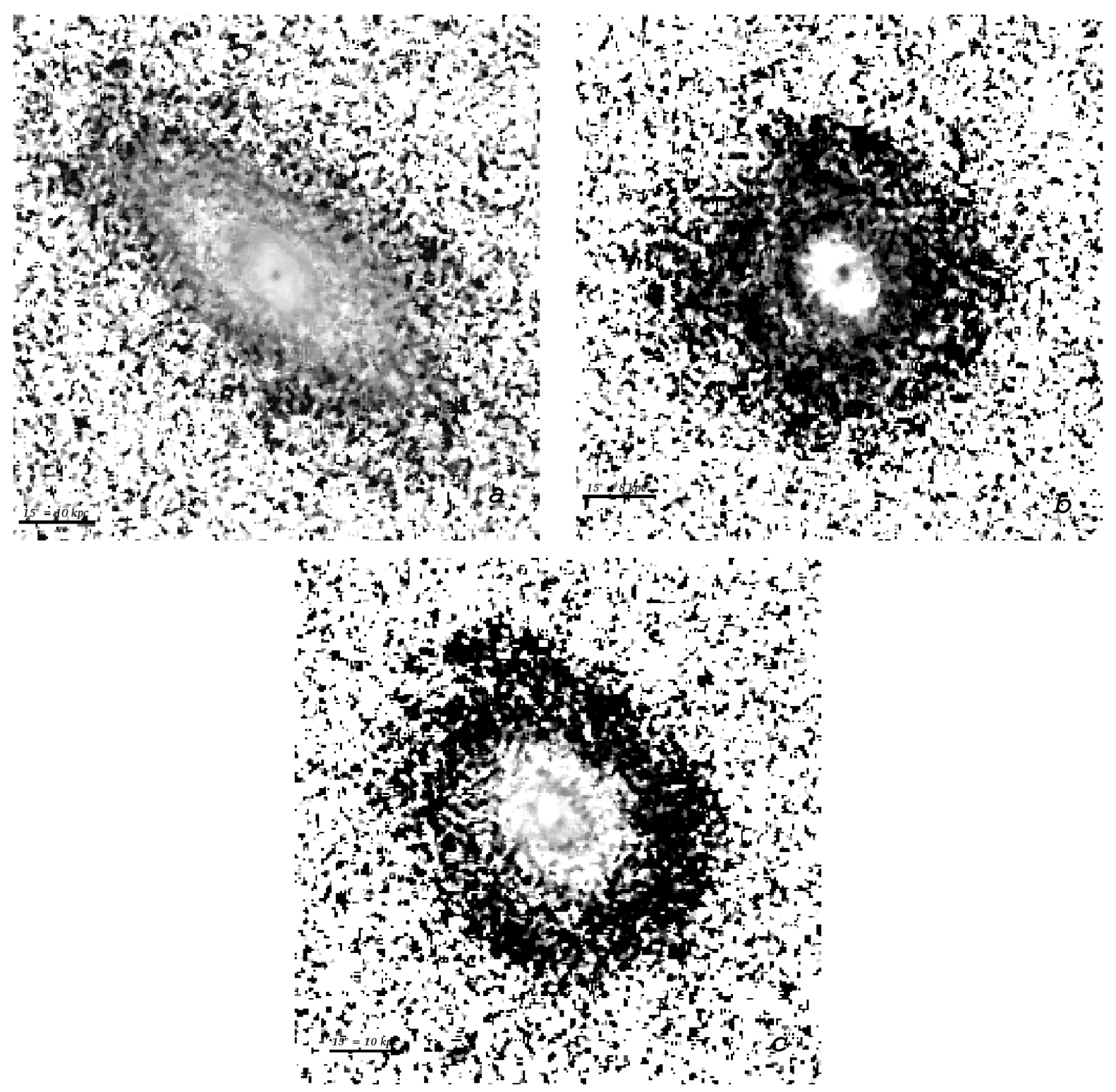

Fig. 5. $B-V$ color maps. North is on top and East to the left. Lower left bar represents $15^{\prime \prime}$ and the corresponding projected scale in Kpc is also indicated. a) ESO 602 - G031, b) ESO 025 - G 002, c) ESO 354 - G 004.

ESO 602-G031: This object is a very luminous SABa $\left(M_{B}=-21.28\right)$. It has the typical integrated colors of normal spirals (see Fig. 1d). The luminosity profiles are well fitted with the Gauss + bulge + disk components. However, an excess above the fitted profile can be noted at $r=5^{\prime \prime}$ due to the presence of the bar, which is less prominent in the $I$ band. The disk of ESO 602-G 031 shows a central cutoff radius of about $10 \mathrm{kpc}$. This feature is well noted in the $B-V$ color map, which is presented in Fig. 5a, showing that the inner $10 \mathrm{kpc}$ are redder ( $\sim 0.3 \mathrm{mag})$ than the outermost regions. The color profiles do not present very pronounced gradients. The outer isophotes show evidence of perturbation, suggesting that the galaxies located to the NE and SW are physical companions. Additional support for this hypothesis can be drawn from the fact that these two galaxies also show signs of tidal interactions.
ESO 025- G002: According to its $B$ image, we classify it as of SAB type. The galaxy is almost face-on, making evident not only the bar but also a ring located at $6 \mathrm{kpc}$ from the nucleus (see Fig. 1e). Along the ring, we detected several HII regions with fluxes lower than $10^{-16} \mathrm{erg} \mathrm{cm}^{-2} \mathrm{~s}^{-1}$. It is interesting to note that this is the only galaxy of the sample that shows extended $\mathrm{H} \alpha$ emission (see Fig. 4). The disk of ESO 025- G002 needs a cutoff radius of $\sim 4 \mathrm{kpc}$ to properly fit the luminosity profile. The $B-V$ color map (see Fig. 5b) and the $B-V$ profile (see Fig. 4b) clearly reveal this effect: a reddened inner region with $r=5 \mathrm{kpc}$. The color of this region averages $(B-V)=1.2$.

1H 1934-063: This galaxy is classified as elliptical in the RC3. However, it shows a very prominent disk and a rather blue color $[(B-V)=0.61]$. Moreover, several arms not well developed are easily observed from our $B$ image (see 
Fig. 1f), leading us to conclude that this objects is of Sb type. Its total radius $(r=4 \mathrm{kpc})$ and its luminosity $\left(M_{B}=-18.74\right)$ indicate that $1 \mathrm{H} 1934-063$ is a rather small galaxy. The luminosity profiles shows an excess over the fitted profile that we interpreted as due to the presence of spiral arms. Luminosity profile decomposition shows that the disk is almost twice as luminous as the bulge. In the $B$ band, the Seyfert nucleus contributes with $20 \%$ of the total luminosity of the galaxy. No extended emission nor possible companions are detected.

1H 2107-097: We classified this galaxy as S0. From Fig. $1 \mathrm{~g}$ a disk and a very faint halo that extents up to $18 \mathrm{kpc}$ from the nucleus can be seen. However, it was not possible to trace the luminosity profile up to this radius due the bright star located to the $\mathrm{W}$ of the galaxy. The derived magnitude for this object up to a radius of $9 \mathrm{kpc}$ is $M_{B}=-20.68$, indicating a rather compact galaxy. The integrated color $(B-V)=0.61$ is typical of a normal spiral. In the $B$ band, the Seyfert nucleus contributes $30 \%$ of the total luminosity. The color profiles show steeper gradients towards the galaxy center. However, in the outermost regions the $B-V$ profile shows a rather constant value while the $V-I$ profile becomes abnormally redder $(\sim 1.8)$. $1 \mathrm{H} 2107-097$ is another sample galaxy that shows a disk with a cutoff radius. The value for this radius is $\sim 3 \mathrm{kpc}$.

ESO354-G004: This galaxy is cataloged in the RC3 as ( $\mathrm{R}^{\prime}: \mathrm{SA}(\mathrm{rs}) \mathrm{b}$. However, no evidence of any ring structure is observed in Fig. 1h. ESO 354-G004 is rather luminous $\left(M_{b}=-21.22\right)$ and its radius extends up to $20 \mathrm{kpc}$. The luminosity profiles are well fitted with a bulge and disk components. The disk shows a cutoff at about $10 \mathrm{kpc}$. This radius coincides with a red $[(B-V)=1.1]$ region that can be observed from its color map in Fig. 5c. This region is also noticeable in the $V-I$ profile. The integrated colors are consistent with an early type stellar population. Signs of tidal interaction are seen in the images. In fact, it is interesting to note that the galaxy located towards the south seems to be connected with ESO 354-G 004 by a very faint arm. On the other hand, the object located towards the SW does not show any visible connection with the main galaxy but the arms of ESO 354-G 004 are perturbed in this direction. These two small galaxies have similar integrated colors, $(B-V) \sim 0.8$.

MRK 509: This galaxy is a very particular object (see Fig. 1i). It is the most luminous and bluest galaxy $\left[M_{B}=\right.$ $-22.34,(B-V)=0.15]$ of our sample. In addition, it shows the largest luminosity contribution of the Seyfert nucleus to the total luminosity $\left(L_{\text {Gauss }} / L_{\text {total }}=0.56\right)$. This fact affects the integrated colors, which are abnormally blue compared with colors of any morphological type. This object is quite well known for being highly variable both in the continuum and emission lines (Peterson et al. 1998). Its relatively small size ( $\sim 10 \mathrm{kpc}$ in radius) makes it also a rather compact object. Apparently, it looks as an elliptical (E1). However, the luminosity profile follows the $r^{1 / 4}$ law up to $r=6.5 \mathrm{kpc}$. Beyond this limit, an excess, which amounts $5 \%$ of the bulge luminosity is well fitted with a disk profile with a cutoff radius of $8.7 \mathrm{kpc}$. It is possible that the galaxy located towards the NW of MRK 509 at about $3^{\prime}$ be a physical companion. In fact, it has a $(B-V)=0.48$ and shows rather disturbed isophotes, giving additional support to this idea.

CTS F10.01: This galaxy is of S0 type and shows a prominent disk (see Fig. 1j). From the luminosity profiles we derived a disk luminosity almost twice as large as that of the bulge. It has $M_{B}=-21.99$ and a radius of $18 \mathrm{kpc}$, indicating that it is a compact object. Despite its morphological type, it is also rather blue $[(B-V)=0.67]$. Luminosity profiles show an excess respect to the fitting in the range $5-8^{\prime \prime}$. This excess is similar in all bands and can be due to the presence of a lens structure.

\section{Conclusions}

We have presented new photometric BVI data for 10 Seyfert 1 galaxies together with narrow band $\mathrm{H} \alpha$ images for 6 of these objects. The absolute $B$ magnitudes of the galaxies, $M_{B}$, are found to be spread over a large interval, from -18.74 to -22.34 . Integrated $(B-V)$ and $(V-I)$ colors as well as morphological types are derived for the first time in most of the objects. We found that the morphologies are confined to early type galaxies: one elliptical, five SO, one Sa and three Sb. Overall, $50 \%$ of the objects can be considered as compacts. Bars are found only in 2 cases $(22 \%)$. The $(B-V)$ colors of the galaxies are shown to be biased to the blue. In fact, the SO galaxies of the sample shows, on average, $(B-V)=0.78$, significantly bluer than the average for this morphological type. We interpret this effect as due to a high contribution of the AGN and/or the disk to the total luminosity of the galaxy.

Signs of tidal interactions are detected in six galaxies of the sample. The case for CTS A08.12 is interesting since it seems to be located in a poor cluster not yet identified in the literature. However, it is not possible to confirm if they are physical interacting objects due to the lack of radial velocities of the suggested companions.

Luminosity profiles were adequately fitted to the Gauss + bulge + disk components. In six out of eight disk models it was necessary to truncate the exponential profile in order to improve the fit. The radius of the central cutoff ranged from 3 up to $10 \mathrm{kpc}$ and it usually corresponds to reddened regions, generally well identified in the $B-V$ color maps. These regions present very similar colors among the sample galaxies, $(B-V) \sim 1.2$. We associate them with the presence of dust in the inner few kiloparsecs of the galaxies.

The profile decomposition allowed us to derive the luminosity contribution of the AGN, bulge and disk separately. We found that in the blue band the AGN contribution to the total luminosity varies from $3 \%$ up to 
$56 \%$. In addition, the bulge to disk ratio ranges from $L_{\text {bulge }} / L_{\text {disk }}=0.6$ to 22 .

$\mathrm{H} \alpha$ images show that only 1 out of 6 galaxies presents disk emission. Additional data is needed in order to confirm whether this emission is photoionized by the nuclear continuum or any starbutst component.

Acknowledgements. We are grateful to Dr. S. Lumsden (referee) for his comments and sugestions.

This work was partially supported by SECyT, PRONEX/FINEP grants 76.97.10003.00 and Fundação de Amparo a Pesquisa do Estado de São Paulo - FAPESP, under contract 00/01020-5.

\section{References}

Barnes, J. E., \& Hernquist, L. E. 1991, ApJ, 370, 65

Chirstensen, C. G. 1975, AJ, 80, 282

de Jong, R. S. 1996, A\&A, 313, 377

De Robertis, M. M., Hayhoe, K., \& Yee, H. K. C. 1998, ApJS, 115,163

de Vaucouleurs, G., de Vaucouleurs, A., Corwin, J. R., et al. 1991, The Third Reference Catalogue of Bright Galaxies (Springer Verlag, New York) (RC3)

González-Delgado, R. M., Pérez, E., Tadhunter, C., Vilchez, J. M., \& Rodríguez-Espinosa, J. M. 1997, ApJS, 108, 155 (GD97)

Ho, L. C., Filippenko, A. V., \& Sargent, L. W. 1997, ApJ, 487, 591

Hunt, L. K., Malkam, M. A., Moriondo, G., \& Salvati, M. 1999, ApJ, 510, 637

Hunt, L. K., Malkan, M. A., Salvati, M., et al. 1997, ApJS, 108,229
Jedrezejewski, R. L. 1987, MNRAS, 226, 747

Kotilainen, J. K., \& Ward, M. J. 1994, MNRAS, 266, 953 (KW94)

Landolt, A. U. 1992, AJ, 104, 372

Malkan, M. A. 1983, ApJ, 268, 582

Márquez, I, Durret, F., Masegosa, J., et al. 2000, A\&A, 360, 431

Maza, J., Ruiz, M. T., Gonzalez, L. E., Wischnjewky, M., \& Antezana, R. 1994, Rev. Mex. Astron. Astrofis., 28, 187

Maza, J., Ruiz, M. T., Gonzalez, L. E., \& Wischnjewky, M. 1992, Rev. Mex. Astron. Astrofis., 24, 147

Maza, J., Ruiz, M. T., Gonzalez, L. E., \& Wischnjewky, M. 1989 ApJS, 69, 349

Pastoriza, M. G., Donzelli, C. J., \& Bonatto, C. 1999, A\&A, 347,55

Peterson, B. M. Wanders, I., Bertram, R., et al. 1998, ApJ, 501, 82

Pogge, R. W. 1989, ApJ, 345, 730 (P89)

Prieto, M., Beckman, J. E., Cepa, J., \& Varela, A. M. 1992, A\&A, 257, 85

Regan, M. W., \& Mulchaey, J. S. 1999, AJ, 117, 2676

Rodríguez-Ardila, A., Pastoriza, M. G., \& Donzelli, C. J. 2000, ApJS, 126, 63 (RPD2000)

Sánchez-Portal, M., Díaz, A. I., Terlevich, R., et al. 2000, MNRAS, 312, 2

Sérsic, J. L. 1982, Extragalctic Astronomy (Reidel, Dordrecht) Shombert, J. M., \& Bothun, G. D. 1987, ApJ, 9260

Tantalo, R., Chiosi, C., Bressan, A., Marigo, P., \& Portinari, L. 1998, A\&A, 335, 823

Terlevich, R., Tenorio-Tagle, G., Franco, J., \& Melnick, J. 1992, MNRAS, 255, 713

Véron-Cetty, M. P., \& Véron, P. 1996, A\&AS, 115, 97

Yee, H. K. C. 1983, ApJ, 272, 473 